AperTO - Archivio Istituzionale Open Access dell'Università di Torino

\title{
LPV approximation of distributed parameter systems in environmental modelling
}

\section{This is the author's manuscript}

Original Citation:

Availability:

This version is available http://hdl.handle.net/2318/6420

since

Published version:

DOI:10.1016/j.envsoft.2004.09.015

Terms of use:

Open Access

Anyone can freely access the full text of works made available as "Open Access". Works made available under a Creative Commons license can be used according to the terms and conditions of said license. Use of all other works requires consent of the right holder (author or publisher) if not exempted from copyright protection by the applicable law. 


\title{
LPV approximation of distributed parameter systems in environmental modelling
}

\author{
Gustavo Belforte $^{\mathrm{a}, *}$, Fabrizio Dabbene ${ }^{\mathrm{b}}$, Paolo Gay ${ }^{\mathrm{c}}$ \\ ${ }^{a}$ Dipartimento di Automatica e Informatica Politecnico di Torino - Corso Duca degli Abruzzi 24, 10129 Torino, Italy \\ bIEITT-CNR Politecnico di Torino - Corso Duca degli Abruzzi 24, 10129 Torino, Italy \\ ${ }^{\mathrm{c}}$ Dipartimento di Economia e Ingegneria Agraria Forestale e Ambientale, Università degli Studi di Torino via Leonardo da Vinci, \\ 44, 10129 Grugliasco (To), Italy
}

Received 19 May 2004; received in revised form 11 July 2004; accepted 23 September 2004

\begin{abstract}
Environmental systems often involve phenomena that are continuous functions not only of time, but also of other independent variables, such as space coordinates. Typical examples are transportation phenomena of mass or energy, such as heat transmission and/or exchange, humidity diffusion or concentration distributions. These systems are intrinsically distributed parameter systems whose description usually requires the introduction of partial differential equations (PDE). Therefore, their modelling can be quite complex, both for what concerns the model construction and its identification. Indeed, a typical approach for the simulation of such systems is the use of finite elements techniques. However, this kind of description usually involves a huge number of parameters and requires time-consuming computations while not being suited for identification. For this reason, such models are generally not suitable for control purposes. In many cases, however, the involved phenomena depend on the independent (space) variables in a smooth way, and for fixed values of the independent variables, input-output relations can be satisfactorily represented by linear time-invariant models. In such conditions, a possible alternative to PDE consists in representing the physical system with a Linear Parameter Varying (LPV) model whose parameters are functions of the independent variables. The advantage of this approach is the relatively simple model obtained, which is directly suitable for control purposes and can be easily identified from input-output data by means of classical techniques. Moreover, optimal identification schemes can be derived for such models, allowing the optimization of the number of measurements. This can be particularly useful in several environmental applications for which the cost of measurements represents a severe constraint.

In this paper, the derivation of LPV models for the representation of distributed phenomena in environmental systems is discussed, and the issue of model uncertainty is addressed. In particular, it is shown that the derived models are linear in the parameters, and therefore classical methods for handling uncertainty are directly applicable. The proposed approach is illustrated by means of a simulated and a practical example concerning soil disinfestation by solarization.
\end{abstract}

(c) 2004 Elsevier Ltd. All rights reserved.

Keywords: Agricultural and environmental processes; Distributed parameter systems; LPV models

\footnotetext{
* Corresponding author. Tel.: +39011564 7018; fax: +39011564 7099.

E-mail addresses: gustavo.belforte@polito.it (G. Belforte), fabrizio.dabbene@polito.it (F. Dabbene), paolo.gay@unito.it (P. Gay).
}

\section{Introduction}

In environmental and agricultural sciences, complex systems need often to be described with mathematical models. The development of model structures adequate for practical use is carried out with different approaches, depending on the goals of the modelling process as well 
as on the available information. Therefore, at one extreme, large simulation models based on the best available knowledge on the involved processes are developed. Such models are often too complex to be satisfactorily identified from experimental data and too detailed to be suitable for control needs. At the other extreme, simple low order input-output "black box" models are derived processing available data with no or little use of a priori knowledge on the involved process. Alternatives and drawbacks of these approaches are nicely discussed in a recent paper by Young and Chotai (2001).

It is well accepted that models to be used in control applications need to be simple and robust to uncertainties; nevertheless, the possibility to incorporate in the model construction some knowledge on the involved process is a desirable feature. Also, the possibility to state a correspondence between the model behavior, its parameters and the physical nature of the real system is often seen as a great advantage.

Most part of environmental and agricultural processes are intrinsically distributed parameter systems, and their behavior is therefore naturally described by partial differential equations (PDE) that, besides being function of time, depend also on spatial coordinates. Possible examples are given by processes in which mass or energy transport phenomena occur. The resulting models are infinite dimensional state models and are, in general, difficult to be identified and managed, even in the simple linear case. Moreover, in many cases, parameter values are separately obtained from specific laboratory tests and not from direct measurements on the system, see for instance, Wu et al. (1996).

Indeed, applying finite-elements techniques it is possible to approximate such models by equivalent finite state models driven by ordinary differential equations in which the infinite number of states is replaced by a large number of states. Finite-elements models are usually presented in discrete-time form to be implemented on digital computers and their large number of states increases with the required accuracy in approximating the original infinite dimensional system. The resulting model is therefore suitable for batch simulations but is far too complex for control purposes.

In control engineering practice, when focus is on input-output relations to be used for control design, high dimensional systems are commonly approximated with low order linear time-invariant (LTI) models. The case of distributed parameter systems regarded as systems with a "large number of states" is not an exception. Hence, low order approximating models can be derived for distributed parameter systems. It is also possible to add some physical interpretation to the different parameters and features of the approximated model. For example, some of its time constants could be regarded as diffusion constants or transport time constants between the input and the output of the distributed parameter real system. Simplified timeinvariant linear parameter models however lose any information about the spatial structure of the original system and cannot account for it although they can be satisfactory from an input-output point of view.

When distributed parameter systems are modelled for monitoring or control purposes, simple input-output models are indeed convenient, but some information about the spatial structures of the system should be possibly preserved. In fact, although in practice the system is usually operated applying specific inputs and measuring corresponding outputs at some particular points of the system volume, nevertheless monitoring and/or control may concern the whole relevant volume of the distributed parameter system and are not restricted to only one or a few pairs of input-output points.

Hence, some simplified model structure that still preserves some information about the spatial structure of the system is needed. Such structure can be provided by Linear Parameter Varying (LPV) models consisting of a linear lumped parameter model in which the parameters are not constant, but are functions of an extra, possibly vector valued, variable $x$ that can be regarded as an input determining the "operating condition" of the model (Rugh and Shamma, 2000; Shamma and Xiong, 1999). The basic idea is the same used for many years in gain-scheduling controllers, see e.g. Leith and Leithead (2000). By selecting the operating condition $x$ to be the spatial coordinate of the inputs and/or outputs of the system some information about the spatial structure can be directly included in the simplified model and hence used to derive results valid throughout the system volume. It should be remarked, however, that such a model remains basically an inputoutput model, able to describe the forced response of the system. Therefore, it is not suited for replacing the infinite dimensional model driven by partial differential equations (or its finite element approximation) when one is interested in simulating the internal behavior of the distributed parameter system.

In this paper, we discuss the use of LPV models for describing distributed parameter systems when information about the spatial structure of the system is needed. In Section 2, the notation and the main ideas of this approach are developed. Different ways for handling errors and uncertainties using the proposed modelling structure are discussed in Section 3. Section 4 reports a simulated example relative to the determination of the temperature in a bar plunged at its extremes in two fluids at different temperatures. In Section 5, the case of soil temperature prediction during a disinfestation process by solarization is discussed and the proposed techniques are applied to real data. Finally in Section 6 conclusions are drawn. 


\section{LPV approximation of distributed parameter systems}

Consider a distributed parameter system subject to an input signal $u(t)$. We are interested in the prediction of the forced response of a distributed variable $y(t ; x)$ to the input signal $u(t)$ for any time $t$ and spatial coordinate $x$. The input is assumed to be concentrated, that is acting at a single spatial point. To this extent we introduce the approximating discrete-time model reported in Fig. 1. This model consists of the cascade of a delay block and an LPV model. Both the delay and the LPV parameters are function of $x$. For the study of such LPV systems with parameter-dependent delays the interested reader should refer to $\mathrm{Wu}$ and Grigoriadis (2001).

The delay block takes into account transport delays in the process and is defined as follows

$\tilde{u}(k ; x)=q^{-\Delta(x)} u(k)$,

where $\Delta(x)$ is an unknown function to be estimated and $q^{-1}$ is the usual unit time shift operator.

The LPV model can have any convenient linear parameter structure in which however the parameters are assumed to be given functions of the spatial coordinate $x$. In the following, we will adopt an autoregressive dynamic structure with exogenous input (ARX) that is quite general and is described by

$A\left(q^{-1} ; x\right) y(k ; x)=B\left(q^{-1} ; x\right) \tilde{u}(k)+e(k)$,

where $e(k)$ takes into account measurement and modelling errors while the regressors $A\left(q^{-1} ; x\right)$ and $B\left(q^{-1} ; x\right)$ are defined as

$$
\begin{aligned}
& A\left(q^{-1} ; x\right)=1+a_{1}(x) q^{-1}+a_{2}(x) q^{-2}+\cdots+a_{n_{a}}(x) q^{-n_{a}} \\
& B\left(q^{-1} ; x\right)=b_{0}(x)+b_{1}(x) q^{-1}+b_{2}(x) q^{-2}+\cdots+b_{n_{b}}(x) q^{-n_{b}}
\end{aligned}
$$

The parameters $a_{i}(x), i=1, \ldots, n_{a}$, and $b_{i}(x), i=0, \ldots, n_{b}$, are continuous functions of the coordinate $x$.

To fully characterize this model, that will be referred to as LPV-ARX $\left(n_{a}, n_{b}\right)$ the functional dependence of the parameters $a_{i}(x)$ and $b_{i}(x)$ on coordinate $x$ must be specified. Possible choices may be polynomials, radial basis functions, splines or other linear combinations of

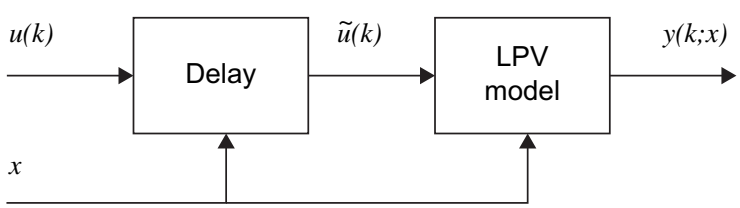

Fig. 1. LPV approximating model for single input distributed parameter systems. given functions of $x$. The whole model can then be identified with a two step approach.

- Step 1: A set of $n$ different suitable values of $x$, $\left\{x_{1}, x_{2}, \ldots, x_{n}\right\}$ is chosen. For any $x_{j}, j=1, \ldots, n$, a set of input-output data is collected. From this data set, an LTI model with the same structure of the LPV model is identified. In this way, $n$ distinct LTI$\operatorname{ARX}\left(n_{a}, n_{b}\right)$ models are obtained, one for each $x_{j}$. Therefore, the parameters of the $j$-th model depend on the value $x_{j}$ used for data set derivation, $a_{i}\left(x_{j}\right)$, $i=1, \ldots, n_{a}, b_{i}\left(x_{j}\right), i=0, \ldots, n_{b}$.

- Step 2: The $n$ different values of the same parameter (e.g. $\left.a_{i}\left(x_{j}\right)\right)$ in the $n$ distinct LTI-ARX $\left(n_{a}, n_{b}\right)$ models derived in Step 1 are used to identify the functions (e.g. $a(x))$ that relate the corresponding parameters of the single LTI-ARX model to the spatial coordinate $x$. These functions may be for instance polynomials or splines. In such a way, LPV parameters are obtained suitably interpolating the parameters of the $n$ LTI-ARX models.

With the resulting LPV model, forecast and control of the output $y(k ; x)$ can be performed for any value of $x$, even different from those used in the identification stage. A similar approach was recently presented in (Coca and Billings, 2002) where the distributed parameter system is approximated with a linear combination of splines weighted with dynamically-varying coefficients.

\section{Uncertainty and error analysis}

The presented modelling and identification procedure, that approximates distributed parameter systems described by partial differential equations with LPV models, leads to point estimates that are convenient for control design. However an error analysis to examine the reliability of the identified model and evidence possible problems is mandatory.

First notice that the LPV-ARX model results to be linear in its parameters whenever the functions $a_{i}(x)$, $i=1, \ldots, n_{a}$ and $b_{i}(x), i=0, \ldots, n_{b}$ are described using generalized polynomials such as B-splines, that is

$a_{i}(x)=\sum_{j=1}^{n_{s}} \alpha_{i, j} \varphi_{j}(x)$

$b_{i}(x)=\sum_{j=1}^{n_{s}} \beta_{i, j} \varphi_{j}(x)$

where $\varphi_{j}(x), j=1, \ldots, n_{s}$ are linear independent continuous functions. In this case, relation (2) rewrites 


$$
\begin{aligned}
y(k ; x)= & \sum_{i=1}^{n_{a}} \sum_{j=1}^{n_{s}} \alpha_{i, j} \varphi_{j}(x) y(k-i ; x) \\
& +\sum_{i=0}^{n_{b}} \sum_{j=1}^{n_{s}} \beta_{i, j} \varphi_{j}(x) \tilde{u}(k-i)+e(k)
\end{aligned}
$$

where $\alpha_{i, j}, i=1, \ldots, n_{a}, j=1, \ldots, n_{s}$ and $\beta_{i, j}, i=0, \ldots, n_{b}$, $j=1, \ldots, n_{s}$ are the parameters to be actually estimated. These parameters can be collected in a single parameter vector

$\theta \in \mathbb{R}^{m}, \quad m=n_{s}\left(n_{a}+n_{b}\right)$,

so that relation (4) can be rewritten as a linear regression model

$y(k)=\Phi \theta+e(k)$.

Indeed, as the model is linear in $\theta$, different techniques are directly applicable for estimating the parameters and their uncertainties. These techniques mainly depend on the different error assumptions. More specifically, if the error $e(k)$ is assumed to be gaussian, it is possible to estimate the parameter-error covariance easily, see for instance Ljung (1987), although this has recognised limitations when the model structure is poor.

A different approach assumes the measurement error $e(k)$ to be unknown but bounded, see for instance Milanese et al. (1996). That is, $e(k)$ is assumed to belong to a membership set

$\Omega_{e}=\{e:|e(k)| \leq E(k), \forall k\}$,

where $E(k)$ is a known bounding function. With this set membership error assumption, the identification of the parameters consists in finding the set $\mathcal{D}$ of all parameters consistent with the model (4), the measurements $y$ and the errors $e$. The corresponding parameter admissible set $\mathcal{D}$ can be expressed as

$\mathcal{D}=\left\{\theta \in \mathbb{R}^{m}: y=\Phi \theta+e, e \in \Omega_{e}\right\}$.

Then, using the techniques described in Milanese et al. (1996), upper and lower bounds on the model parameters $\alpha_{i, j}, \beta_{i, j}$ can be derived.

In the next sections, we present a simulated and a realworld example, showing how the approach proposed in this paper can be efficiently applied. In the examples presented, the error noise was assumed to be gaussian, and the lumped LTI-ARX models used in Step 1 of the LPV identification procedure were identified using the MATLAB Identification Toolbox (Ljung, 1994).

\section{LPV approximation: a simulated case}

In this section we present the simple problem of nonsteady thermal conduction in a one dimensional body. We consider the case of an aluminium bar of length $l=100 \mathrm{~cm}$ plunged at its extremes into two fluids at different temperatures. We assume the temperature $T(t$; $x), x \in[0, l]$, to be the output and the temperature $T_{F 1}(t)$ of the first fluid to be the input. The temperature $T_{F 2}(t)$ of the second fluid is assumed to be constant.

Let the body be initially at a uniform temperature $T_{0}$. The general conduction equation, for uniform and isotropic materials with no internal heat generation, is driven by the following partial differential equation, see for instance (Chapman, 1984),

$\frac{1}{\alpha} \frac{\partial T(t ; x)}{\partial t}=\frac{\partial^{2} T(t ; x)}{\partial x^{2}}$

that is associated with the following initial and boundary conditions

$$
T(0 ; x)=T_{0}, \quad \forall x \in[0, l]
$$

$-k\left(\frac{\partial T(t ; x)}{\partial x}\right)_{x=0}=h\left(T(t ; x)-T_{F_{1}}(t)\right)_{x=0} \quad \forall t>0$

$-k\left(\frac{\partial T(t ; x)}{\partial x}\right)_{x=l}=h\left(T(t ; x)-T_{F_{2}}(t)\right)_{x=l} \quad \forall t>0$,

where $k=204 \mathrm{~W} / \mathrm{m} \cdot \mathrm{K}$ is the thermal conductivity of aluminium, $h=0.562 \mathrm{~W} / \mathrm{m}^{2} \cdot \mathrm{K}$ is the thermal conductance of the two fluids and $\alpha=8.41810^{-5} \mathrm{~m}^{2} / \mathrm{s}$ is the thermal diffusivity.

When a suitable input (temperature of the first fluid) $T_{F_{1}}(t)$ is applied to the system, the simulation of the system of relation (7) allows the temperature output $T(t$; $x$ ) in any section $x$ of the bar to be derived. Such simulation has been performed in this paper by numerical integration using the implicit scheme described in (Jaluria and Torrace, 1986) and (Sewell, 1988).

If a specific section at $x=x^{*}$ is considered, the input-output behavior can be approximated with a time-invariant lumped parameter model derived with any suitable criterion, see e.g. (Ljung, 1987).

The derived LTI model accounts quite well for the temperature in $x^{*}$ also for input signals different from the one used for identification. However, the obtained results do not give any information about the temperature in any other section different from $x^{*}$. Therefore, whenever the goal is to monitor or to control the temperature in any section of the bar, the use of this LTI model is inadequate.

To represent with a simplified model the considered distributed parameter process still preserving some information about the spatial structure of the system, a LPV-ARX model is then constructed.

It is assumed that the process can be well represented by a LPV-ARX $(3,3)$ model whose seven parameters $a_{i}(x) i=1, \ldots, 3$ and $b_{i}(x) i=0, \ldots, 3$ are function of the $x$ 
spatial variable representing the position of the section in which the output is located. The functions relating the parameters to the $x$ variable are assumed to be cubic splines obtained interpolating the parameters of four $\operatorname{ARX}(3,3)$ LTI models. These are identified using $\left\{T_{F 1}(t), T(t ; x)\right\}$ data with $x$ at the four values $\{10 \mathrm{~cm}$, $30 \mathrm{~cm}, 50 \mathrm{~cm}, 70 \mathrm{~cm}\}$, obtained by integrating the partial different equation (Eq. (7)).

Using the resulting LPV-ARX(3,3) model, the temperatures in other three sections $x=\{20 \mathrm{~cm}$, $40 \mathrm{~cm}, 60 \mathrm{~cm}$ \} not used for identification are then computed when the input is a square wave with varying period. Fig. 2 shows the simulation of the identified LPV model at the three sections $x=\{20 \mathrm{~cm}, 40 \mathrm{~cm}, 60 \mathrm{~cm}\}$ (dotted lines), together with the temperatures $T(t ; x)$ computed in the same sections integrating, again, the partial differential equation (Eq. (7)) (solid line). As it can be seen the approximation is quite good and supports the use of such LPV models for keeping spatial information. Indeed, the LPV model output tracks are faster than the distributed parameter system outputs.

Considering the whole range of parameter variations, the obtained LPV model allows the temperature in all the sections of the bar to be monitored. Therefore, a SISO robust controller can be designed that, handling all the possible parameter values, guarantees specifications on the whole volume.

\section{LPV approximation: a real case}

In this section, we present an agricultural application that is quite close to the previously simulated process. In intensive agriculture the continuous exploitation of the

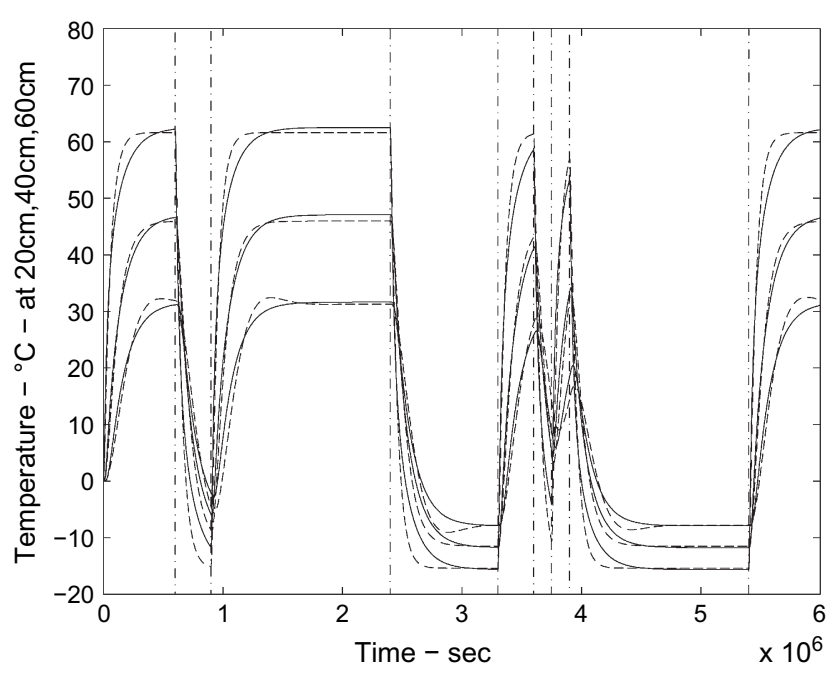

Fig. 2. Validation of the LPV approximation model at the sections $x=20 \mathrm{~cm}, 40 \mathrm{~cm}, 60 \mathrm{~cm}$ : solid line the distributed parameter system output for the single input distributed parameter systems, dotted line the output of the corresponding identified LPV model. soil with the same crop leads to the development of weed seeds, nematodes and plant pathogens. It is therefore necessary to periodically treat the soil in order to kill these undesired agents (Mulder, 1979).

Soil solarization is a procedure used for controlling a wide spectrum of soilborne pathogens and weeds (Katan and DeVay, 1991; Stapleton, 2000). It is performed mulching tilled and irrigated soil with transparent polyethylene sheeting. Soil mulching can be done with a continuous film covering on the whole plot applied either manually or by laying separate strips which are melded together, depending on the technology available. The soil is heated by solar irradiation and soilborne pests are killed due to physical and biological processes.

Here temperature monitoring and prediction is of interest for soil disinfestation. The measured data $\{u(t), y(t ; x)\}$ used in this paper are relative to the solar radiation $\left(\mathrm{W} / \mathrm{m}^{-2}\right)$ and the temperatures $\left({ }^{\circ} \mathrm{C}\right)$ at different depths in the soil, respectively. The polyethylene mulch was laid in August 2002 and removed in September 2002. Data were collected during the complete treatment period and then split into two distinct identification and validation sets. Except for a gradient of reduced effectiveness of solarization toward the edge of the plastic mulch known as border effect (Grinstein et al., 1995), the soil can be macroscopically approximated with an unbounded uniform body. The only dimension that affects the system behavior is the depth $x$ at which temperature is considered.

Temperatures were measured with a probe constituted by six thermocouple placed at depths $x=\{15 \mathrm{~mm}$, $40 \mathrm{~mm}, 65 \mathrm{~mm}, 90 \mathrm{~mm}, 115 \mathrm{~mm}, 140 \mathrm{~mm}\}$. The corresponding data are reported in Fig. 3. In this case an LPV-ARX $(4,4)$ was considered for representing the data. The parameter dependence from the depth $x$ was assumed once more to be represented by cubic splines that have been derived from the data relative to $x=\{15 \mathrm{~mm}, 40 \mathrm{~mm}, 90 \mathrm{~mm}, 115 \mathrm{~mm}, 140 \mathrm{~mm}\}$. The information relative to depth $x=65 \mathrm{~mm}$ was left out in the identification process.

Since the proposed approach aims at deriving a model that enables to forecast the distributed parameter system behavior at any point in space and in particular at those points where measurements were not collected for system identification, the validation procedure was carried out comparing simulated model output data with real data collected at a point (depth $x=65 \mathrm{~mm}$ ) not used for identification. Moreover data sets used for identification and for validation were collected at different times to allow an even better validation. The LPV-ARX $(4,4)$ parameter values for $x=65 \mathrm{~mm}$ have been computed and the model simulated. Real and simulated data are presented in Fig. 4 for a time interval of 12 days. In Fig. 5 the difference between real and 

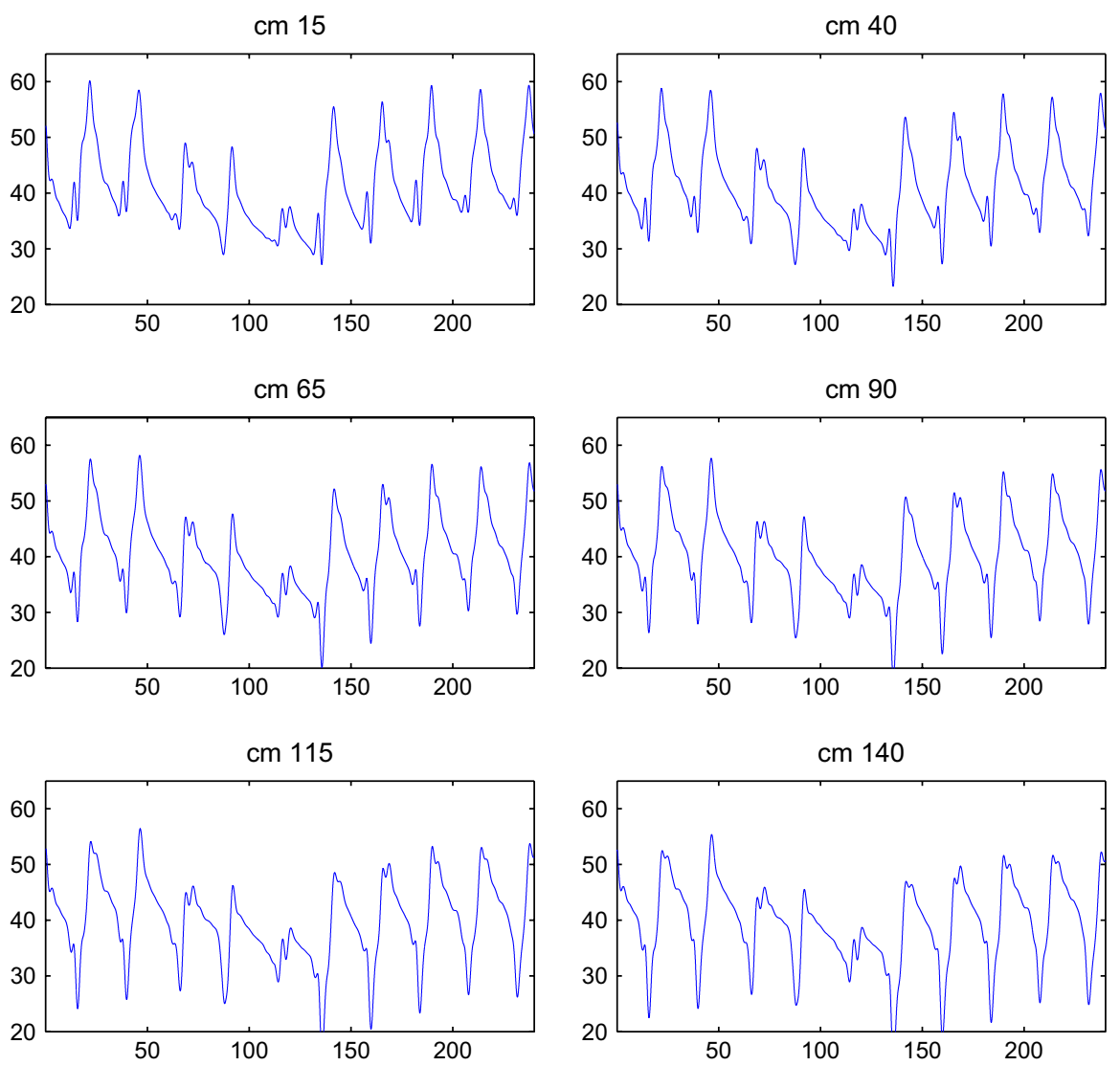

Fig. 3. Temperature of the soil at depths $x=\{15 \mathrm{~mm}, 40 \mathrm{~mm}, 65 \mathrm{~mm}, 90 \mathrm{~mm}, 115 \mathrm{~mm}, 140 \mathrm{~mm}\}$ during a 12 days solarization treatment used for identification purposes. The information relative to depth $x=65 \mathrm{~mm}$ was left out in the identification process.

simulated data (the error) is reported. The signals reported in these plots present several important features that may provide interesting information for a better understanding of the experiment, suggesting also possible future activity.

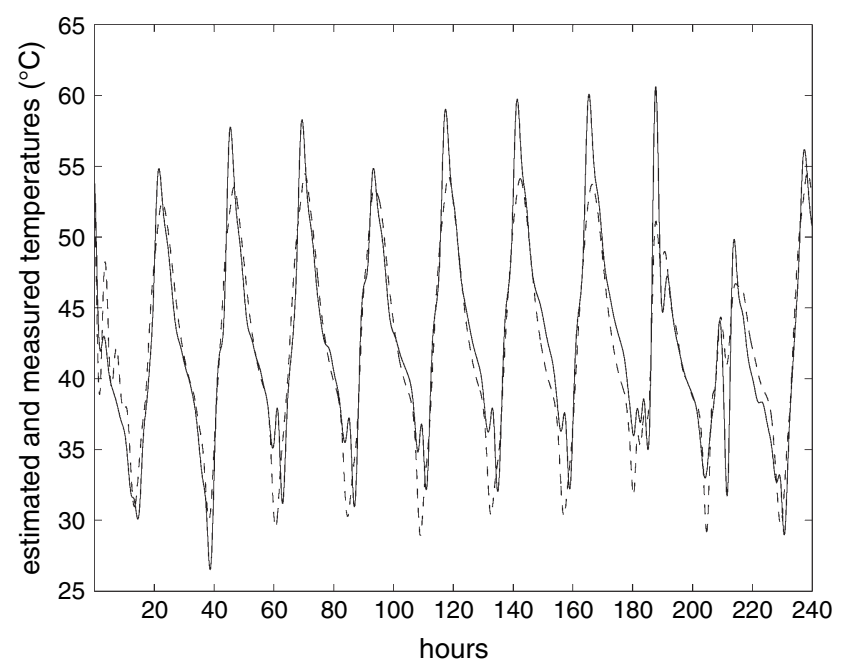

Fig. 4. Temperature of the soil at depth $x=65 \mathrm{~mm}$ during a disinfestation process by solarization: measured data (solid line) and simulated data (dotted line).
- The measured signal presents in general one maximum and one minimum peak a day. They are related respectively to the heating during the day and the cooling during the night. However in most days there is an extra peak appearing at the cooling time.

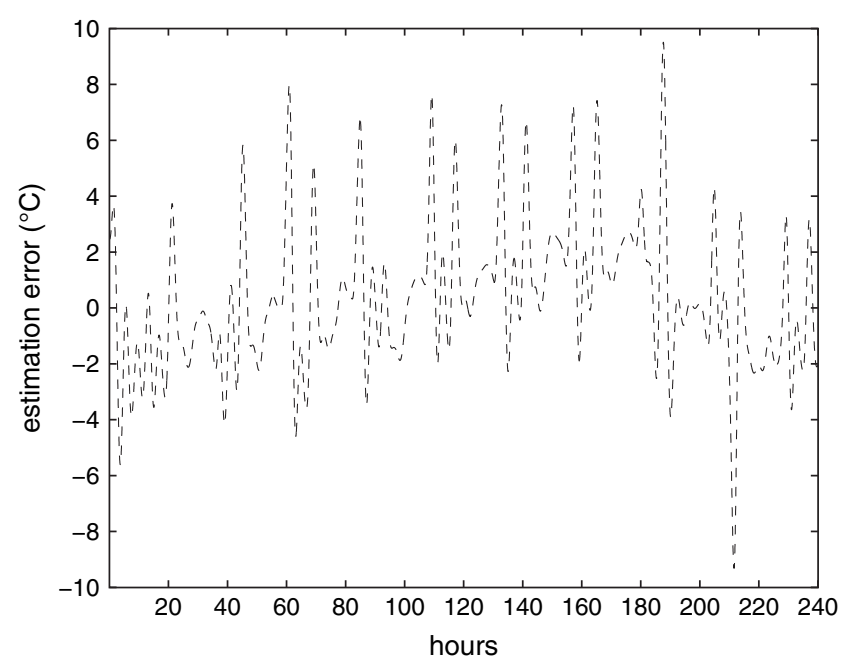

Fig. 5. Error (difference between measured and simulated data) of the LPV-ARX $(4,4)$ model at depth $x=65 \mathrm{~mm}$. 
- The error signal presents some evident periodicity.

- The error has usually a peak close to the peak values of the measured signal.

The most important observation that follows from the previous remarks is that the used input-output model explains the data to a large extent, but does not account for all the output behavior. In particular the extra peak that is present almost every day during the cooling time is not accounted for. The explanation that we can suggest for this systematic error, reconsidering the whole experimental setting, is related to the fact that measurements have been obtained using thermocouple whose cold joint is mainly associated with the data logger collecting the data. Such device was located on the field, covered by a small shelter, that, however perhaps did not properly cover the equipment from direct sun rays during the last hours of the day. It is therefore possible that the temperature of the cold joint was affected by direct sun rays at sunset. This could explain the peak in the data signal during the cooling time. Moreover the fact that in some days the phenomenon does not occur could be simply motivated by the absence of direct sun rays on the device in cloudy days. Unfortunately the available weather record is not sufficient to validate this hypothesis. The variation in temperature of the cold joint could determine also the large errors occurring close to the peaks of the data signal when the overall temperature change could affect again the cold joint. Indeed the assumed model input is the sun radiation only that was measured through a proper transducer that obviously could not account for the changes in temperature of the cold joint. All the previous considerations could also motivate the periodicity present in the error signal.

It must be noted that the problems evidenced before are related to the experimental setting therefore data used for model identification and for model validation are affected likewise. It follows therefore that the minimization of the $l_{2}$ norm of the interpolation error that was used as criterion in the parameter identification stage, is more convenient than the $l_{\infty}$ norm since this last is far more sensitive to local errors.

\section{Conclusions}

This paper introduces a fairly new approach for approximate modelling of intrinsically distributed parameter processes using Linear Parameter Varying (LPV) models.

The use of LPV models allows the system to be approximated with a lumped low order model that however still keeps some information about the spatial structure of the original system. In fact, the parameters of the LPV model are assumed to be functions of the relevant spatial coordinates that account for the distributed parameter nature of the real process. In this way the model, that remains intrinsically an inputoutput model, can be used for monitoring and control solutions that take into account the whole relevant range of the spatial coordinates of the real process.

This kind of approach can be quite valuable in environmental and agricultural applications where distributed parameter processes such as those related to the diffusion of substances and/or to the transmission of heat are quite common. Simple models for such kind of systems are needed for practical applications in which computing time is usually constrained. Moreover, the linear in the parameters structure presented in this paper easily permit to find optimal identification procedures that allow the system parameters to be derived with the smallest possible uncertainty still using a moderate amount of measurements, whose collection is usually expensive in environmental applications.

Beside the above benefits, the proposed approach still presents some problems. In particular, a study of the modelling errors introduced by the procedure should be carried on. Such errors are mainly related to the approximation of a distributed parameter system with a lumped parameter model and to the interpolation process performed whenever outputs are computed for locations different from the ones where measurements were collected.

\section{Acknowledgements}

This work was supported by Research grants of Politecnico di Torino, by IEIIT-CNR, by grant CNR n.99.01960.CT07 and MIUR MM07157922.

\section{References}

Chapman, A., 1984. Heat Transfer. Macmillan Publishing Company, New York.

Coca, D., Billings, S., 2002. Identification of finite dimensional models of infinite dimensional dynamical systems. Automatica 38, 1851-1865.

Grinstein, A., Kritzman, G., Hetzroni, A., Gamliel, A., Mor, M., Katan, J., 1995. The border effect of soil solarization. Crop Protection 14 (4), 315-320.

Jaluria, Y., Torrace, K., 1986. Computational Heat Transfer. Springer-Verlag, New York.

Katan, J., DeVay, J., 1991. Soil Solarization. CRC Press, Boca Raton, USA.

Leith, D., Leithead, W., 2000. Survey of gain-scheduling analysis and design. International Journal of Control 73, 1001-1025.

Ljung, L., 1987. System Identification: Theory for the User. PrenticeHall, Engewood Cliffs, NJ.

Ljung, L., 1994. MATLAB Identification Toolbox. The Mathworks.

Milanese, M., Norton, J., Piet-Lahanier, H., Walter, E. (Eds.), 1996. Bounding approaches to system identification. Plenum Press, New York, USA.

Mulder, D., 1979. Soil Disinfestation. Elsevier Scientific Public Company, Amsterdam. 
Rugh, W., Shamma, J., 2000. Research on gain scheduling. Automatica 36, 1401-1425.

Sewell, G., 1988. The Numerical Solution of Ordinary and Partial Differential Equations. Academic Press, Inc., San Diego.

Shamma, J., Xiong, D., 1999. Set-valued methods for linear parameter varying systems. Automatica 35, 1081-1089.

Stapleton, J., 2000. Soil solarization in various agricultural production systems. Crop Protection 19, 837-841.
Wu, F., Grigoriadis, K., February 2001. LPV systems with parametervarying time delays: analysis and control. Automatica 37 (2), 11-21.

Yihua, W., Perry, K.B., Ristaino, J.B., 1996. Estimating temperature of mulched and bare soil from meteorological data. Agricultural and Forest Meteorology 81 (3), 299-323.

Young, P., Chotai, A., October 2001. Data-based mechanistic modeling, forecasting, and control. IEEE Control Systems Magazine 21 (5), 14-27. 